\title{
An addendum on "Robust control of robots by the computed torque method"*
}

\author{
Harry Berghuis ${ }^{a}$, Henk Nijmeijer ${ }^{b}$, \\ Peter Löhnberg ${ }^{\text {a }}$ \\ ${ }^{a}$ Department of Electrical Engineering, ${ }^{b}$ Department of Ap- \\ plied Mathematics, University of Twente, Enschede, Netherlands
}

Received 20 August 1991

Revised 6 December 1991

Abstract: We reinterprete and improve recent results on robust control of robots by the computed method. The methods and ideas used are inspired by 'passivity based' control methods for robot manipulators and lead to a significant increase in freedom of controller implementation, thereby providing more flexibility to the designer of robot control systems.

Keywords: Robotics; tracking control; unknown dynamics; robustness; Lyapunov stability.

\section{Introduction}

In the last decades a lot of attention has been paid to trajectory tracking control methods for robot manipulators which has led to the development of various different robot control schemes. Roughly, these schemes can be divided into two classes, depending on the way the ProportionalDerivative (PD) feedback part comes into the controller output.

The first class consists of various forms of computed torque control [2,3]. This method achieves the objective of trajectory tracking by feedback linearization of the nonlinear robot dynamics and results in a linear error equation for the closed-loop system, which can be shown to be exponentially stable [10]. Characteristic for computed torque control is that the PD part is multi-

Correspondence to: Dr. H. Berghuis, Department of Electrical Engineering, University of Twente, P.O. Box 217, $7500 \mathrm{AE}$ Enschede, Netherlands.

* This work is supported by the Netherlands Technology Foundation (STW). plied with the time-varying inertia matrix, before it is added to the controller output.

The second class consists of control methods with a PD feedback part that is added directly to the controller output $[1,4,5,7-10]$. To this linear PD part a compensation part for the nonlinear robot dynamics is added. Because the robot dynamics define a passive input/output mapping which by these control methods is preserved in closed-loop, we will refer to them as passivity based control methods [4]. It is important to notice that due to the linearity in the PD part, the error dynamics of a robot system controlled by a passivity based method are in general nonlinear. This shows a main difference with the computed torque method.

In the recent, interesting paper [6] entitled "Robust control of robots by the computed torque method" a robust version of the computed torque controller has been proposed; that is, the exact knowledge of the system dynamics is replaced by a suitable estimate for this dynamics, yielding uniform ultimate boundedness of the tracking error dynamics under a list of suitable assumptions. However, as will be demonstrated in Section 2, under the assumptions made by the authors the proposed controller of [6] is no longer a computed torque controller in case the system dynamics are known. On the other hand, we will show in the same section that this controller belongs to the second class of control methods for trajectory control, i.e. the controller is a robust version of the well-known passivity based control method of Slotine and Li [8].

In Section 3 another, more important, result by relating techniques from literature on passivity based robot control methods is given. We show that some of the earlier restrictive assumptions required in the stability proof of [6] can be removed and thus more design freedom in the controller implementation results, which is of course extremely useful from a practical point of 
view. Section 4 contains the conclusions of this note.

\section{Robust control method in the ideal case}

We briefly recapitulate the notations and problem formulation as given in [6]. Consider the robot dynamics

$M(q) \ddot{q}+N(q, \dot{q})=T$,

$N(q, \dot{q})=V_{\mathrm{m}}(q, \dot{q}) \dot{q}+G(q)+F(\dot{q})$,

where $q$ is an $n \times 1$ vector of joint variables, $M(q)$ is an $n \times n$ (symmetric and positive definite) inertia matrix, $V_{\mathrm{m}}(q, \dot{q})$ is an $n \times n$ matrix of centripetal and Coriolis terms, $G(q)$ is an $n \times 1$ vector of gravity terms and

$F(\dot{q})=F_{\mathrm{d}} \dot{q}+F_{\mathrm{s}}(\dot{q})+T_{\mathrm{d}}$

is the sum of friction and disturbance torques.

For the system (1) the following control law is proposed in [6]:

$T=M_{\mathrm{c}}\left(\ddot{q}_{\mathrm{d}}+K_{\mathrm{v}} \dot{E}+K_{\mathrm{p}} E\right)+N_{\mathrm{c}}$,

where $E=q_{\mathrm{d}}-q$ is the tracking error, $q_{\mathrm{d}}$ represents the desired trajectory and $M_{\mathrm{c}}$ and $N_{\mathrm{c}}$ represent the computed value of $M(q)$ and $N(q, \dot{q})$ respectively. In the ideal case, i.e. $M_{\mathrm{c}}=M(q)$ and $N_{\mathrm{c}}=N(q, \dot{q}),(2)$ represents the computed torque controller. Due to imperfect knowledge of the robot dynamics, $M(q)$ and $N(q, \dot{q})$ are generally now known exactly, and a robust controller can be used. The choice for $N_{\mathrm{c}}$ in [6] is given by

$$
\begin{aligned}
N_{\mathrm{c}}= & N_{\mathrm{k}}+M_{\mathrm{k}} \ddot{q}_{\mathrm{d}}-M_{\mathrm{c}} \ddot{q}_{\mathrm{d}} \\
& +\alpha M_{\mathrm{k}} \dot{E}+\alpha V_{\mathrm{m}, \mathrm{k}} E+V_{\mathrm{m}, \mathrm{k}} \dot{E}
\end{aligned}
$$

where $M_{\mathrm{k}}, N_{\mathrm{k}}$ and $V_{\mathrm{m}, \mathrm{k}}$ represent the known parts of $M(q), N(q, \dot{q})$ and $V_{\mathrm{m}}(q, \dot{q})$ respectively. With the choice (3) the control input (2) can be rewritten as

$$
\begin{aligned}
T= & M_{\mathrm{k}}\left(\ddot{q}_{\mathrm{d}}+\alpha \dot{E}\right)+V_{\mathrm{m}, \mathrm{k}}(\dot{E}+\alpha E) \\
& +N_{\mathrm{k}}+s_{2} \dot{E}+s_{1} E
\end{aligned}
$$

where also the gains $M_{\mathrm{c}} K_{\mathrm{v}}$ and $M_{\mathrm{c}} K_{\mathrm{p}}$ have been replaced by the positive scalars $s_{2}$ and $s_{1}$ respectively (see the assumption made in the appendix of $[6])$.
Proposition 1. The robust controller (4) does not feedback linearize the robot system (1) in the case that the system dynamics are known exactly.

Proof. If the system dynamics are known exactly, then

$$
M_{\mathrm{k}}=M(q), \quad V_{\mathrm{m}, \mathrm{k}}=V_{\mathrm{m}}(q, \dot{q}), \quad N_{\mathrm{k}}=N(q, \dot{q}) .
$$

As a consequence, (4) becomes

$$
\begin{aligned}
T= & M(q)\left(\ddot{q}_{\mathrm{d}}+\alpha \dot{E}\right)+V_{\mathrm{m}}(q, \dot{q})\left(\dot{q}_{\mathrm{d}}+\alpha E\right) \\
& +G(q)+F(\dot{q})+s_{2} \dot{E}+s_{1} E .
\end{aligned}
$$

The error dynamics that describe the closed-loop system (1)-(6) are given by

$$
\begin{aligned}
& M(q)(\ddot{E}+\alpha \dot{E})+V_{\mathrm{m}}(q, \dot{q})(\dot{E}+\alpha E) \\
& \quad+s_{2} \dot{E}+s_{1} E=0 .
\end{aligned}
$$

So the closed-loop system is governed by a nonlinear error equation. This shows that the ideal version (6) of the robust law (4) does not feedback linearize the system.

The 'ideal' control law (6) does not correspond to the true computed torque method. Firstly, the compensation for $V_{\mathrm{m}}(q, \dot{q})$ in (6) differs from the compensation for $V_{\mathrm{m}}(q, \dot{q})$ in the computed torque method. Secondly, the PD feedback part is directly added to the controller output $T$, causing the error dynamics (7) to be nonlinear. In order to obtain the linear error equation that is characteristic for the computed torque method, it is necessary to have $M_{\mathrm{c}}$ in (2) equal to $M(q)$. But this will make $s_{1}, s_{2}$ time-varying, which significantly increases the complexity of the stability analysis because $s_{1}, s_{2}$ are contained in the Lyapunov function $V(x)$; see (4) in [6].

A closer analysis of [6] shows more striking aspects. Firstly, the stability proof is based on a Lyapunov function that represents a modified energy function. Secondly, the skew-symmetric property of $\dot{M}(q)-2 V_{\mathrm{m}}(q, \dot{q})[4,6]$ is utilized in the proof. Both aspects, together with the fact that the PD part is directly coming into (6), are characteristic for the class of passivity based control methods for robot systems [1,7-10]. This indicates that the presented controller belongs to this class, which indeed turns out to be the case. 
Proposition 2. Assume that the robot dynamics (1) are known. Then the robust controller (4) is a particular case of the adaptive passivity based control method presented in [8].

Proof. Consider the non-adaptive version of the adaptive control method presented in [8]. For the robot system (1) this controller is given by

$$
\begin{aligned}
T= & M(q)\left(\ddot{q}_{\mathrm{d}}+\alpha \dot{E}\right)+V_{\mathrm{m}}\left(\dot{q}_{\mathrm{d}}+\alpha E\right) \\
& +G(q)+F(\dot{q})+K_{\mathrm{d}} S
\end{aligned}
$$

where $S=\dot{E}+\alpha E$. The controller (8) is equivalent to the one given in (6) by choosing $\alpha K_{\mathrm{d}}=s_{1} I$, $K_{\mathrm{d}}=s_{2} I$.

Proposition 2 implies that the control method (4) represents a robust version of the (non-adaptive) controller of [8], in which the unknowns $M(q), V_{\mathrm{m}}(q, \dot{q}), N(q, \dot{q})$ have been replaced by their known parts $M_{\mathrm{k}}(q), V_{\mathrm{m}, \mathrm{k}}(q, \dot{q}), N_{\mathrm{k}}(q, \dot{q})$. This shows that (4) actually belongs to the class of passivity based control methods.

We would like to mention that, by utilizing the skew-symmetric property, the part $V_{\mathrm{m}, \mathrm{k}} S$ in the controller (4) compensates for the part $S^{\mathrm{T}} \dot{M}_{\mathrm{k}}(q) S$ in the Lyapunov derivative, see appendix $A$ in [6]. However, it should be noticed that $S^{\mathrm{T}} \dot{M}_{\mathrm{k}}(q) S$ can be upper bounded by a third order term in the state variable $x$, and consequently can be included in $\|\Delta A\|$. This implies that the use of the skew-symmetry is not necessary, and that $V_{\mathrm{m}, \mathrm{k}} S$ can actually be removed from the control input (4).

\section{Generalization of stability proof by a state transformation}

The error dynamics describing the closed-loop system (1)-(4) are given by

$$
\begin{aligned}
& M(q) \dot{S}+V_{\mathrm{m}, \mathrm{k}}(q, \dot{q}) S+s_{2} \dot{E}+s_{1} E \\
& \quad=M_{\mathrm{u}}\left(\ddot{q}_{\mathrm{d}}+\alpha \dot{E}\right)+N_{\mathrm{u}} .
\end{aligned}
$$

In [6] stability for this closed-loop system is shown with the Lyapunov function

$$
V(x)=\frac{1}{2} x^{\mathrm{T}}\left[\begin{array}{cc}
\left(s_{1}+\alpha s_{2}\right) I+\alpha^{2} M(q) & \alpha M(q) \\
\alpha M(q) & M(q)
\end{array}\right] x
$$

where $x^{\mathrm{T}}=\left[E^{\mathrm{T}}, \dot{E}^{\mathrm{T}}\right]$. This function can be rewritten as

$$
\begin{aligned}
V(x)= & \frac{1}{2}(\dot{E}+\alpha E)^{\mathrm{T}} M(q)(\dot{E}+\alpha E) \\
& +\frac{1}{2}\left(s_{1}+\alpha s_{2}\right) E^{\mathrm{T}} E \\
= & \frac{1}{2} S^{\mathrm{T}} M(q) S+\frac{1}{2}\left(s_{1}+\alpha s_{2}\right) E^{\mathrm{T}} E
\end{aligned}
$$

where $S=\dot{E}+\alpha E$. This type of Lyapunov function, which can be considered as a modified energy function, is standard in passivity based robot control literature $[1,7,9]$.

Both the error dynamics (9) and the Lyapunov function (11) suggest the introduction of a new state, defined as $y^{\mathrm{T}}=\left[E^{\mathrm{T}}, S^{\mathrm{T}}\right]$ (see also [9]). This new state vector is related to $x$ via a linear state space transformation $y=W x$, where

$W=\left[\begin{array}{cc}I & 0 \\ \alpha I & I\end{array}\right]$.

With this new state and some modifications in the control law (4) and the Lyapunov function (11), the stability proof as presented in [6] can be given under considerably less restrictive assumptions. This is shown in the following.

First of all, some upper bounds on the unknowns $N_{\mathrm{u}}, V_{\mathrm{m}, \mathrm{u}}$ expressed in the new state $y$ have to be determined. Similar to [6], such upper bounds can be given by

$$
\begin{aligned}
& \left\|N_{\mathrm{u}}\right\| \leqslant \beta_{0}+\bar{\beta}_{1}\|y\|+\bar{\beta}_{2}\|y\|^{2}, \\
& \left\|V_{\mathrm{m}, \mathrm{u}}\right\| \leqslant \beta_{3}+\bar{\beta}_{4}\|y\|,
\end{aligned}
$$

where $\bar{\beta}_{i}, i=1,2,4$, are functions of $\alpha$, and $\beta_{0}$, $\beta_{3}$ as defined in [6].

Next consider the following modified version of the control law (4) (see also [7]):

$$
\begin{aligned}
T= & M_{\mathrm{k}}\left(\ddot{q}_{\mathrm{d}}+\alpha \dot{E}\right)+V_{\mathrm{m}, \mathrm{k}}(\dot{E}+\alpha E) \\
& +N_{\mathrm{k}}+\bar{K}_{\mathrm{v}} \dot{E}+\left(\alpha \bar{K}_{\mathrm{v}}+\bar{K}_{\mathrm{p}}\right) E \\
= & M_{\mathrm{k}}\left(\ddot{q}_{\mathrm{d}}+\alpha \dot{E}\right)+V_{\mathrm{m}, \mathrm{k}}(\dot{E}+\alpha E) \\
& +N_{\mathrm{k}}+\bar{K}_{\mathrm{v}} S+\bar{K}_{\mathrm{p}} E
\end{aligned}
$$

where $\bar{K}_{\mathrm{p}}, \bar{K}_{\mathrm{v}}$ are positive definite symmetric matrices. The difference with (4) is that the scalars $s_{1}, s_{2}$ have been replaced by matrices $\alpha \bar{K}_{\mathrm{v}}+\bar{K}_{\mathrm{p}}$, $\bar{K}_{\mathrm{v}}$ respectively. Choose the following Lyapunov function:

$$
V^{*}(y)=\frac{1}{2} S^{\mathrm{T}} M(q) S+\frac{1}{2} E^{\mathrm{T}} \bar{K}_{\mathrm{p}} E .
$$


Then the lemma given in [6] can be modified as follows.

Lemma. The Lyapunov function (15) satisfies the following inequalities:

$\bar{\lambda}_{\mathrm{p}}^{\mathrm{L}}\|y\|^{2} \leqslant V^{*}(y) \leqslant \bar{\lambda}_{\mathrm{p}}^{\mathrm{U}}\|y\|^{2}$,

$\dot{V}^{*}(y) \leqslant\|y\|\left(\xi_{0}-\bar{\xi}_{1}\|y\|+\bar{\xi}_{2}\|y\|^{2}\right)$,

where

$\bar{\lambda}_{\mathrm{p}}^{\mathrm{L}}=\min \left(m_{1}, \mu_{\mathrm{p}}^{\mathrm{L}}\right), \quad \bar{\lambda}_{\mathrm{p}}^{\mathrm{U}}=\max \left(m_{2}, \mu_{\mathrm{p}}^{\mathrm{U}}\right)$,

$\bar{s}_{3}=\min \left(\alpha \mu_{\mathrm{p}}^{\mathrm{L}}, \mu_{\mathrm{v}}^{\mathrm{L}}\right)$,

$\mu_{\mathrm{p}}^{\mathrm{U}}=\max \left\|\bar{K}_{\mathrm{p}}\right\|, \quad \mu_{\mathrm{p}}^{\mathrm{L}}=\min \left\|\bar{K}_{\mathrm{p}}\right\|$,

$\mu_{\mathrm{v}}^{\mathrm{L}}=\min \left\|\bar{K}_{\mathrm{v}}\right\|$,

$\xi_{0}=\beta_{0}+\lambda_{3} m_{3}, \quad \bar{\xi}_{1}=\bar{s}_{3}-\beta_{3}-\bar{\lambda}_{2} m_{3}-\bar{\beta}_{1}$,

$\bar{\xi}_{2}=\bar{\beta}_{4}+\bar{\beta}_{2}$,

$\bar{\lambda}_{2}=\lambda_{\max }\left(\bar{R}_{2}\right), \quad \bar{R}_{2}=\frac{1}{2}\left[\begin{array}{cc}0 & -\alpha^{2} I \\ -\alpha^{2} I & 2 \alpha I\end{array}\right]$,

and $m_{1}, m_{2}, m_{3}, \lambda_{3}$ as defined in [6].

Proof. Inequality (16a) follows directly from (15). Next, the closed-loop system (1)-(14) is given by (compare with (9))

$$
\begin{aligned}
& M(q) \dot{S}+V_{\mathrm{m}, \mathrm{k}}(q, \dot{q}) S+\bar{K}_{\mathrm{v}} S+\bar{K}_{\mathrm{p}} E \\
& \quad=M_{\mathrm{u}}\left(\ddot{q}_{\mathrm{d}}+\alpha \dot{E}\right)+N_{\mathrm{u}} .
\end{aligned}
$$

The time-derivative of $V^{*}(y)$ in (15) along (17) can be shown to be

$$
\begin{aligned}
\dot{V}^{*}(y)= & -S^{\mathrm{T}} \bar{K}_{\mathrm{v}} S-\alpha E^{\mathrm{T}} \bar{K}_{\mathrm{p}} E \\
& +S^{\mathrm{T}}\left(M_{\mathrm{u}}\left(\ddot{q}_{\mathrm{d}}+\alpha \dot{E}\right)+N_{\mathrm{u}}+V_{\mathrm{m}, \mathrm{u}} S\right)
\end{aligned}
$$

where the skew-symmetric property has been used. $V^{*}(y)$ can be rewritten by

$$
\begin{aligned}
\dot{V}^{*}(y)= & -y^{\mathrm{T}} Q y+y^{\mathrm{T}}\left[\begin{array}{l}
0 \\
I
\end{array}\right] V_{\mathrm{m}, \mathrm{u}}\left[\begin{array}{l}
0 \\
I
\end{array}\right]^{\mathrm{T}} y \\
& +y^{\mathrm{T}} \bar{R}_{2}\left[\begin{array}{cc}
M_{\mathrm{u}} & 0 \\
0 & M_{\mathrm{u}}
\end{array}\right] y+y^{\mathrm{T}}\left[\begin{array}{l}
0 \\
I
\end{array}\right] \Delta A
\end{aligned}
$$

where $\Delta A=M_{\mathrm{u}} \ddot{q}_{\mathrm{d}}+N_{\mathrm{u}}$ as defined in [6], and

$Q=\left[\begin{array}{cc}\alpha \bar{K}_{\mathrm{p}} & 0 \\ 0 & \bar{K}_{\mathrm{v}}\end{array}\right]$.

Using (13) an upper bound for $V^{*}(y)$ is given by (16b).

The representation of the system (1)-(14) in this new state $y$ makes that the Lyapunov function $V^{*}(y)$ as given in (15) is positive definite in $y$ for all $\alpha>0$. This shows that the restriction $0<\alpha<1$ as determined in [6] with the Gershgorin Theorem is not necessary for this redefined state. Because $\alpha$ in some sense determines the convergence rate of the tracking error $E$, cf. [8], this is especially from a practical point of view a useful advantage. However, it should be noted that the meaning of $\alpha$ may be slightly different here, as convergence of the tracking error is not necessarily guaranteed with the robust controllers (4) and (14).

A second advantage is that the highly restrictive assumption that the PD part is completely determined by the two scalars $s_{1}, s_{2}$, see (4), is not needed. In [6] this restriction seems to be introduced in order to simplify the use of the Gershgorin Theorem, which is not needed here. The modified lemma has been proven for the controller (14) which contains PD feedback gains given by the positive definite matrices $\alpha \bar{K}_{\mathrm{v}}+\bar{K}_{\mathrm{p}}$ and $\vec{K}_{v}$ respectively. This significantly increases the flexibility in the controller implementation.

There is one point that needs some further attention. The constants $\bar{\beta}_{i}, i=1,2,4$, are dependent on $\alpha$. In particular, $\bar{\beta}_{1}$ and $\bar{\beta}_{4}$ are linear in $\alpha$, whereas $\bar{\beta}_{2}$ is quadratic in $\alpha$. In the limiting case that $\alpha=0$ the equality $\bar{\beta}_{i}=\beta_{i}, 1=1,2,4$, holds. However, notice that this dependency is not really causing a problem because $\alpha$ is just a design parameter.

Based on the lemma we obtain the following two theorems.

Theorem 1. The error system (17) is stable in the sense that it is uniformly ultimately bounded if $\bar{s}_{3}$ is chosen to be large enough.

Proof. The proof can be given using the same techniques as in the proof of Theorem 2 in [6], 
but now based on the results of the lemma as given in this note.

Theorem 2. If the final value of the desired trajectory is an equilibrium point, i.e.

$\ddot{q}_{\mathrm{d}}, \dot{q}_{\mathrm{d}} \rightarrow 0$, and $q_{\mathrm{d}} \rightarrow \bar{q}$ as $t \rightarrow \infty$

where $\bar{q}$ is a constant, if the static balancing torque is known, i.e.

$G_{k}(\bar{q})=G(\bar{q})$

where $G_{k}$ denotes the known part of $G$, and if $F(\dot{q}) \equiv 0$, then there is a finite gain $\bar{s}_{3}$ for which the error system is asymptotically stable.

Proof. Similar to the proof of Theorem 3 in [6].

Notice that with respect to Theorem 3 in [6] we make the additional assumption that $F(\dot{q}) \equiv 0$ (or at least is should be known precisely so that it can be compensated for) because in the presence of for example Coulomb friction, asymptotic stability of the closed-loop system can not be guaranteed for a finite gain $\bar{s}_{3}$.

\section{Conclusion}

In this note it has been shown that the robust control law presented is [6] does not belong to the class of computed torque controllers due to the fact that the control output is linear in the PD feedback part, which results in a nonlinear error equation for the closed-loop system. On the contrary, the presented law [6] possesses some typical features that are characteristic for passivity based robot control methods. We have proven that the control law is a robust version of a well known passivity based control method [8].

With ideas that are known from stability proofs in passivity based robot control literature, it has been shown that some restrictive assumptions that have been made by the authors of [6] can be removed.

\section{References}

[1] H. Berghuis, P. Löhnberg and H. Nijmeijer, Adaptive 'PD +' control of rigid robot manipulators, Proc. IFAC Symp. on Robot Control, Vienna, Austria (1991).

[2] J.J. Craig, Introduction to Robotics - Mechanics and Control (Addison-Wesley, Reading, MA, 1986).

[3] J.Y.S. Luh, M.W. Walker and R.P.C. Paul, Resolved acceleration control of mechanical manipulators, IEEE Trans. Automat. Control 25 (1980) 468-474.

[4] R. Ortega and M.W. Spong, Adaptive motion control of rigid robots: a tutorial, Automatica 25 (1989) 877-888.

[5] B. Paden and R. Panja, Globally asymptotically stable 'PD +' controller for robot manipulators, Internat. J. Control 47 (1988) 1697-1712.

[6] Z. Qu, J.F. Dorsey, X. Zhang and D.M. Dawson, Robust control of robots by the computed torque method, Systems Control Lett. 16 (1991) 25-32.

[7] N. Sadegh and R. Horowitz, Stability and robustness analysis of a class of adaptive controllers for robotic manipulators, Internat. J. Robotics Res. 9 (1990) 74-92.

[8] J.-J.E. Slotine and W. Li, On the Adaptive Control of Robot Manipulators, Internat. I. Robotics Res. 6 (1987) 49-59.

[9] M.W. Spong, R. Ortega and R. Kelly, Comments on "Adaptive manipulator control: A case study", IEEE Trans. Automat. Control 35 (1990) 761-762.

[10] J.T. Wen and D.S. Bayard, New class of control laws for robotic manipulators: Non-adaptive case, Internat. J. Control 47 (1988) 1364-1386. 